\title{
Capacidad de uso de las tierras en la microcuenca El Limón,' complejo de cuencas Sabana Yegua, República Dominicana
}

\author{
Land use capacity in the mini watershed El Limón, Sabana \\ Yegua Watershed Complex, Dominican Republic
}

Maldané Cuello Espinoza²

Fecha de recepción: I I de abril del 2012

Fecha de aprobación: 16 de agosto del 2012

Cuello, M. Capacidad de uso de las tierras en la microcuencia El Limón, complejo de cuencas Sabana Yegua, República Dominicana. Tecnología en Marcha. Vol. 26, N I. Pág 77-85

Basado en Cuello, M. 2009. Plan Estratégico para el manejo de las tierras en la microcuenca del arroyo El Limón, Cuencas Altas Sabana Yegua, República Dominicana. Avance Tesis de Doctorado. UNA, CR.

2 Especialista en Manejo de Cuencas Hidrográficas. Tel. (829) 9861600. Correo electrónico: maldanecuello@gmail.com 


\section{Palabras clave}

Capacidad de uso de la tierra, prácticas de conservación de suelos, sistemas forestales, sistemas agroforestales, erosión, clases de tierras.

\section{Resumen}

Este estudio aborda el análisis biofísico de las tierras en la microcuenca El Limón, en el complejo de Cuencas Altas de Sabana Yegua en República Dominicana. El objetivo del ensayo fue determinar la capacidad de uso de las tierras considerando las limitaciones del suelo, el clima y la geomorfología que presenta. Se propone una serie de prácticas de manejo como opciones para reducir la erosión, mejorar el suelo y acondicionar los caminos, lo que permitiría mantener condiciones favorables para el manejo racional del sistema. De esa forma, con este objetivo se establecen y se integran por primera vez en una cuenca del país varios elementos limitantes para con su identificación proponer prácticas apropiadas que controlen el problema de degradación acelerada que experimenta el área bajo estudio. Para ello, se determinaron parámetros fundamentales, los cuales abarcan factores de erosión, suelo, drenaje y clima, en función de lo que establece la metodología MAG/FAO/UNED (1996), la cual se usa en Costa Rica para determinar la capacidad de uso de las tierras y ha sido adaptada al área objeto de este estudio. Esta metodología integra clases de tierras que van desde la I hasta la VIII según la frecuencia y grado de las limitantes que se identifican. Para su aplicación en la microcuenca El Limón, se han incluido otros parámetros físicos que no contemplan dicha metodología, tales como la densidad aparente del suelo y los parámetros climáticos de lluvia y temperatura, estos últimos con el fin de establecer la zona de vida del área.

Se pudo determinar que las clases de tierras en la microcuenca El Limón van desde la III hasta la VIII, las cuales son aptas para protección, sistemas forestales y agroforestería. De igual manera, se estableció que las mayores limitantes que se presentan son las concernientes a la erosión y al drenaje del suelo. Para frenar y corregir los efectos de estas limitantes, las prácticas de manejo recomendadas son las de labranza mínima, rotación de cultivos, uso de barreras muertas con residuos de cosecha, barreras vivas con el uso de material biológico de la zona y terrazas individuales en sistemas agroforestales. Estas prácticas son alternativas para que los productores continúen desarrollando los sistemas de producción que tradicionalmente utilizan, con un criterio técnico de manejo.

\section{Key words}

Land use capacity, soils conservation practices, forestry systems, agroforestry systems, land classes parameters.

\section{Abstract}

This study conduced the biophysical analysis of El Limón micro watershed within the high land Sabana Yegua watershed, Dominican Republic. The objective of the research was directed to determine its land capacity use, taking into account limitations of soils, climate and geomorphology. It proposes a series of management practices as options to reduce erosion, improve soil and roads conditions, in order to get better conditions for a rational system management. In this way, for reaching such objective for the first time some basic elements could be established and integrated to deal with the accelerated degradation problem affecting the area under study. For getting this purpose fundamental parameters were established, such as factors related to erosion, soil, drainage and climate, in function of the methodology MAG/ FAO/UNED (1996) used in Costa Rica to determine land capacity use, which has been adapted to the area under study. This methodology has been used as the basic tool in this essay, integrating land classes from class I to class VIII in acordance with the frecuency and level limitations identified. For its application in the Limón micro watershed, we included other physical parameters that were not observed in this methodology, such as soil apparent density and the climatic parameters, such as rain and temperature daily registers, the last two in order to determine the life zone.

The research established that the land classes in EI Limón micro watershed include land from class III to VIII. All land classes are able for protection, forestry systems and agroforestry. In the same manner, it could be established that major limitations are related to erosion and soil drainage. For making corrections to these limitations, the recommended practices are minimal tillage, crops rotation, and use of dead barriers with harvest residuos, live barriers with natural biological products of the zone and individual terraces in agroforestry systems. These practices allow producers to develop their traditional system production with a technical criteria management. 


\section{Introducción}

La microcuenca El Limón se encuentra ubicada dentro de un complejo de cuencas consideradas prioritarias en el ámbito nacional, por la importancia hidrológica que representa para toda la región sur del país.

Sin embargo, la microcuenca y su conjunto experimentan un gran deterioro ambiental causado por el mal manejo de las tierras, prácticas de producción inadecuadas y las malas condiciones de sus caminos de acceso.

Una de las principales consideraciones del estudio es determinar cómo formular técnicas para el uso apropiado de los recursos de la zona que puedan ser adoptadas por los productores, y que involucren aspectos para mejorar los mismos dentro de sus sistemas de producción sostenible con el enfoque de cuencas.

Mediante el estudio se determinó la capacidad de uso de las tierras en la microcuenca El Limón, con el objetivo de detectar las limitaciones biofísicas y climáticas que se presentan y establecer las prácticas más apropiadas dentro de las unidades de manejo determinadas.

Se pudo evidenciar que las mayores limitaciones de las tierras en la microcuenca son de suelo, erosión y drenaje, lo que podría ser la causa de la poca rentabilidad de los sistemas agrícolas tradicionales.

También se determinó que las clases de tierra, según las unidades de manejo son básicamente de protección con vocación forestal, para lo cual se recomiendan prácticas conservacionistas, como son los sistemas agroforestales con café (Coffea arábica), aguacate (Persea americana) y limón (Citrus limonium). De igual manera, en función de las limitantes mencionadas, para el establecimiento de sistemas agrícolas se han propuesto prácticas agroconservacionistas que ayudarían a restaurar el suelo, favorecen el drenaje excesivo y controlan la erosión, como es el uso de barreras muertas, barreras vivas y terrazas individuales.

\section{Materiales y métodos}

El área que comprende la microcuenca El Limón abarca una superficie de 7/32 hectáreas (ha), correspondiente a un importante afluente tributario del río Grande o del Medio, siendo este último uno de los tres que componen el gran sistema de cuencas altas de Sabana Yegua, al sur del país (figura. 2).

La determinación de las características físicoquímicas del suelo, la descripción geomorfológica y el análisis del clima comprenden el desarrollo metodológico

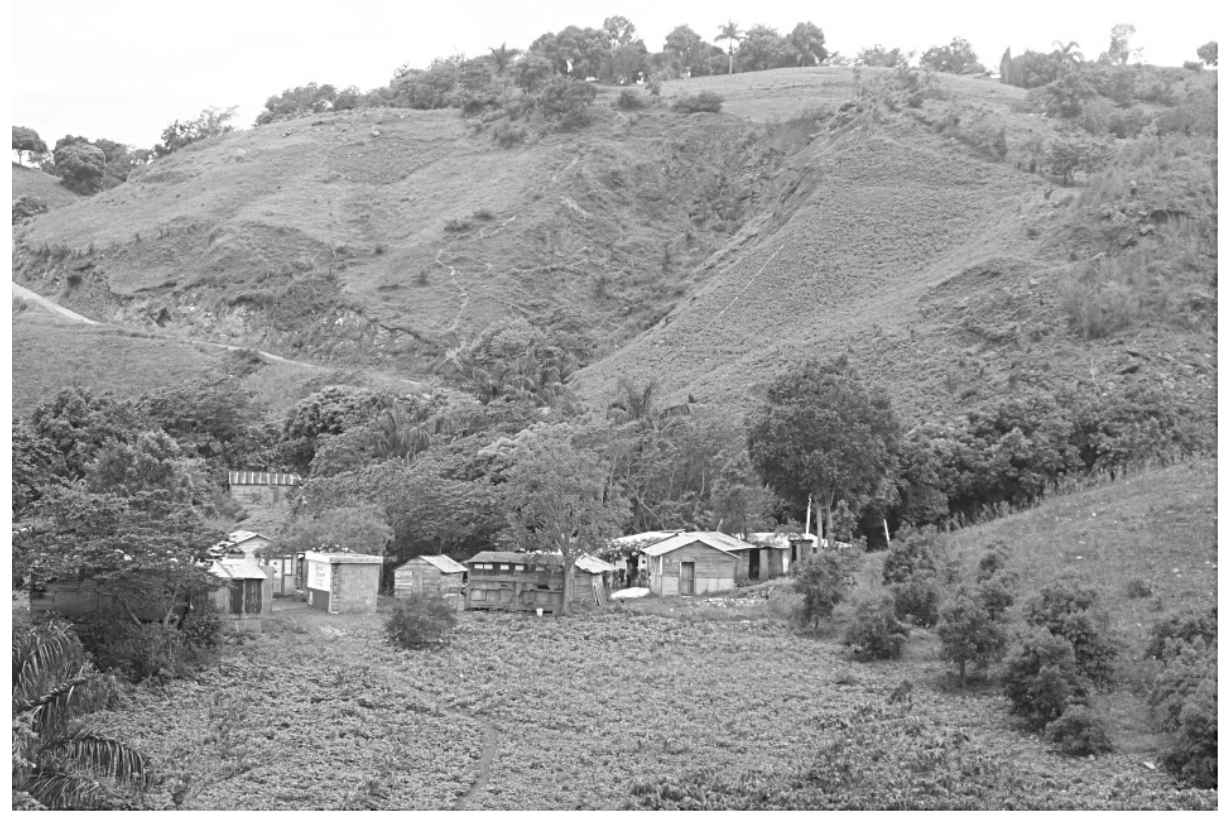

Figura I. Aspecto general de la microcuenca El Limón, República Dominicana. 201 I. 


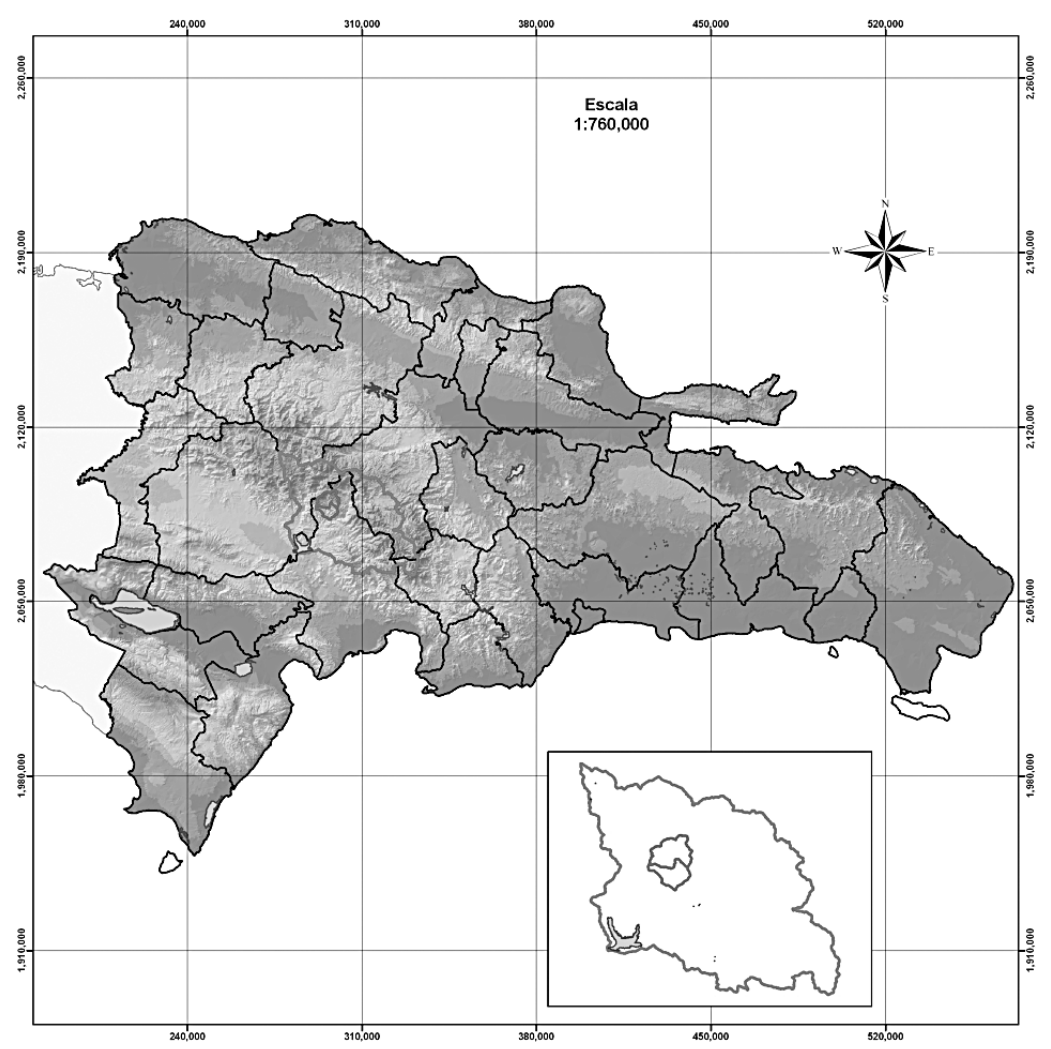

Figura 2. Ubicación de la microcuenca El Limón en el contexto nacional.

para conocer la capacidad de uso de las tierras, así como para establecer las prácticas agrícolas y de conservación de suelo más apropiadas. Cada uno de dichos parámetros se determinó in situ y en laboratorio, para así obtener los insumos o datos requeridos.

La estructura del sistema de clasificación de las tierras comprende tres niveles: ocho clases, subclases y unidades de manejo, este último indica el factor o los factores específicos que limitan su utilización en actividades agropecuarias y forestales (Cubero, 2008). Para los fines de su aplicación en la microcuenca El Limón, se determinaron previamente nueve Unidades Fisiográfica (UF) en un grid obtenido de una imagen de satélite reciente, a escala I: 60000. La delimitación de cada UF obedeció a la fisiografía natural del terreno según la pendiente exhibida, tal como se observa en el mapa de la figura 3. Además de levantar, obtener y analizar las informaciones y datos requeridos por la metodología, se determinó la densidad aparente del suelo en cada UF; este aspecto se detalla en el parámetro erosión; así como categorías o rango de parámetros de zona de vida, enfocándose este aspecto en el parámetro clima.

\section{Parámetros para la evaluación de la capacidad de uso de las tierras}

I. De erosión (e). Para conocer las limitantes que presentan las tierras en estos términos, se determinaron los parámetros de pendiente y de la erosión sufrida en el suelo in situ. El primero, de pendiente (I), pudo establecerse con el uso de un clinómetro, un aparato que mide la pendiente en porciento entre dos puntos equidistantes del observador y a diferentes alturas. El segundo parámetro: de erosión sufrida (2), se determinó de acuerdo con la presencia en el campo de evidencias del proceso de erosión, como son la cantidad y magnitud de pedestales, de surcos y cárcavas en el terreno. Las categorías de ambos parámetros se establecen en el manual usado para la aplicación del sistema de clasificación.

2. De suelo (s). Este factor se refiere al estado del recurso al integrar parámetros que determinan su estado o limitación. Los parámetros determinados para su evaluación son de profundidad efectiva del suelo (I), 


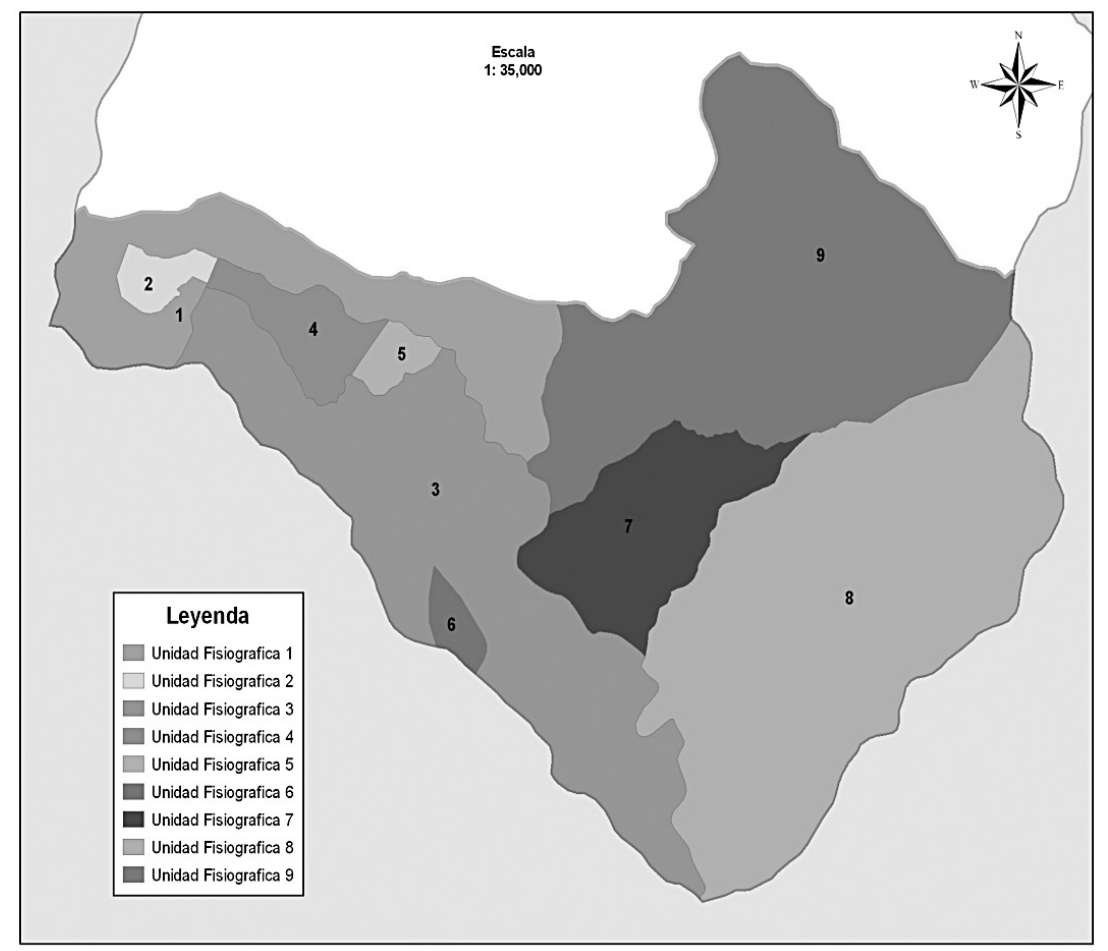

Figura 3. Mapa de las Unidades Fisiográficas. Fuente: creación propia.

comprendida hasta donde las raíces pueden penetrar sin dificultad -para lo cual se realizaron perfiles típicos en cada unidad fisiográfica-, de textura (2), tanto del suelo como del subsuelo, para ello se tomaron muestras extraídas de los horizontes de los perfiles realizados y se enviaron al laboratorio para su análisis; pedregosidad y/o rocosidad (3), este parámetro, al igual que la erosión, se determina de acuerdo al porcentaje de material rocoso reinante tanto dentro como fuera del perfil de suelo, las categorías de la pedregosidad se indican en el manual para la clasificación; fertilidad (4), su determinación depende de la acidez del suelo y su capacidad de intercambio catiónico, para lo cual las muestras de suelo se enviaron al laboratorio; toxicidad de cobre (5), son los niveles de cobre que tiene el suelo, su determinación se hizo a nivel de laboratorio, al igual que la salinidad (6), siendo ésta indicada por la concentración total de sales en el suelo; densidad aparente (7), cuya determinación depende del suelo seco que cabe en un volumen establecido, sus valores indican la capacidad del suelo para la retención de humedad. Este parámetro se determinó en el laboratorio de física de suelos, secando las muestras tomadas con un volumen conocido durante 24 horas en un horno a $105^{\circ} \mathrm{C}$; para su determinación se usó la siguiente fórmula:

$$
\mathrm{Da}=\mathrm{P} / \mathrm{g}\left(\mathrm{g} / \mathrm{cm}^{3}\right)
$$

$$
\begin{array}{lll}
\text { (ecuación I) } & V=\pi r^{2} h ; \\
\text { Donde: } & \pi=3.1416
\end{array}
$$

$\mathrm{P}=$ Peso seco de la muestra $(\mathrm{g}) \mathrm{r}^{2}=$ radio, el cual es de $(\mathrm{d} / 2)^{2}$.

$V=$ Volumen de la muestra $\left(\mathrm{cm}^{3}\right) \mathrm{h}=$ largo de molde toma muestra

4. De drenaje (d). Rapidez de desplazamiento del agua tanto a nivel superficial como a lo interno del suelo. Este parámetro se determinó in situ, tratándose de la evidencia del grado - magnitud de ocurrencia. Las categorías para este parámetro se detallan en el manual usado para la clasificación y pueden ser: excesivo, moderadamente excesivo, bueno, moderadamente lento, lento, muy lento y nulo; este último no se consideró en el estudio pues no es aplicable según las condiciones de irregularidad del terreno. 
5. De clima (c). Según la metodología MAG/FAO/ UNED, este factor involucra la determinación de los parámetros de zonas de vida según la clasificación de Holdridge, como el factor (I), período seco (2), presencia de neblina (3) y categoría de viento (4), siendo los dos últimos determinados in situ. No se muestran valores por la ausencia considerable de ellos en la zona estudiada. Sin embargo, para el parámetro de zonas de vida fue necesaria la búsqueda de registros de promedios mensuales de lluvia y de temperatura del área de la microcuenca, los cuales, al promediar los registros de seis años consecutivos, indican que el promedio mensual de lluvia al año es de 978 mm y el promedio mensual de la temperatura es de $21{ }^{\circ} \mathrm{C}$. De igual manera, la clasificación de Holdridge requiere el valor de la altitud, este dato se determinó durante sondeos para la toma de muestras de suelo, el cual va desde 750 hasta 1400 metros sobre el nivel del mar (msnm). Al integrar estos datos de clima, la clasificación indica que la zona pertenece al Bosque Húmedo Montano Bajo (bh-MB). Esta zona de vida coincide con la determinada para toda el área de las cuencas altas Sabana Yegua por la Compañía PROYECTA en 2007.

En relación con el período seco, este es el número consecutivo de meses secos en el año, entendiéndose como meses secos aquellos donde la precipitación es inferior a la mitad de la evapotranspiración potencial. La evapotranspiración potencial se determinó multiplicando los promedios de temperatura mensuales por una constante establecida en el manual de clasificación, a saber: 4,54 para meses

Cuadro I. Clases y unidades de manejo de tierras

\begin{tabular}{|c|c|c|c|}
\hline UF & Clase & Factores limitantes & Unidad de manejo \\
\hline I & V & es d & Vel2 s234 dl \\
\hline 2 & VI & e sd & $\mathrm{VI}$ el$\underline{2} \mathrm{~s} / 3 \mathrm{dl}$ \\
\hline 3 & VIII & esd & VIII el2 s $\_23 \mathrm{dl}$ \\
\hline 4 & III & es d & III el$\underline{2}$ sl $\underline{2} \mathrm{dl}$ \\
\hline 5 & IV & es $d$ & IV e $12 \mathrm{~s} \mid 2 \underline{3} \mathrm{dl}$ \\
\hline 6 & $\mathrm{VI}$ & e sd & $\mathrm{VI}$ el2 s $123 \mathrm{dl}$ \\
\hline 7 & $\mathrm{VI}$ & esd & $\mathrm{VI}$ el2 sl23 dl \\
\hline 8 & VII & es d & VII el2 sl23 dl \\
\hline 9 & VII & esd & $V|l e \perp 2 s| 23 \mathrm{dl}$ \\
\hline
\end{tabular}

Fuente: creación propia. de 28 días, 4,84 para meses de 30 días y 5,00 para meses de 31 días. Cuando la mitad de estos valores de promedio mensual de evapotranspiración es mayor que el promedio de lluvia mensual, se consideran meses secos, determinándose que en la zona hay tres meses secos consecutivos (enero, febrero y marzo), por lo que su categoría corresponde a período seco moderado. Para obtener las unidades de manejo, se utilizó como herramienta el programa computacional Sistema de Diagnóstico de Tierras Tropicales (200I).

\section{Descripción de las prácticas}

Esta descripción se refiere a las acciones o intervenciones que corresponde desarrollar en cada una de las unidades fisiográficas, para enfrentar los parámetros limitantes determinados en las unidades de manejo. Estas prácticas de manejo, conservación de suelo y agua, implican la utilización de material biológico vivo o muerto, materia inerte y la participación de los productores.

Las prácticas recomendadas se presentan en el cuadro I y son las que se aplican según las limitaciones y la severidad con que las mismas se manifiestan. En este sentido, se presenta la readecuación de los caminos como técnica de conservación de suelo. Esta técnica ha implicado realizar un levantamiento topográfico en varios tramos para determinar sus pendientes más típicas, las cuales se establecieron midiendo en el terreno los tramos más críticos, tomando las coordenadas de sus puntos extremos y determinando su altura de acuerdo con las curvas de nivel de una hoja topográfica de la zona a escala 1:50 000. La pendiente se determinó dividiendo la diferencia de altura de los puntos extremos entre la distancia existente entre ambos, expresada en porciento; pudiéndose determinar tramos con pendientes que oscilan entre 0\%-15\%, 15\%-30\% y mayor de $30 \%$. Con estas denominaciones de pendiente, se realizaron tres diseños para cada una de ellas, integrando elementos que sirvan para controlar la escorrentía, canalización de agua fluvial, drenaje natural y la protección de taludes.

\section{Resultados y discusión}

\section{Capacidad de uso de las tierras}

La capacidad de uso de las tierras se fundamentó en los parámetros determinados que involucran los elementos de erosión, suelo, drenaje y clima, de los cuales solo el clima no resultó ser un factor 
limitante, por lo que las nueve unidades fisiográficas necesitan tratamientos que controlen o regulen los procesos que envuelven los parámetros limitantes. Las clases de tierras en la microcuenca abarcan desde la III hasta la VIII (cuadro I).

Según la capacidad de uso de las tierras, existen limitantes de erosión (e), de suelo (s) y de drenaje (d), siendo tierras aptas para la implementación de sistema forestal y de protección, sin embargo, considerando el impacto económico negativo que significaría mantener el área cubierta solo con estos dos sistemas, se han establecido algunas prácticas agroconservacionistas y de conservación de suelo que permitan controlar el efecto de la degradación, al mismo tiempo que los productores pueden aprovechar sus predios y mantener sus sistemas agrícolas tradicionales: habichuela (Faseolus vulgaris) y guandul (Cajanus cajans).
Todas las unidades de manejo muestran limitaciones de pendiente $\left(e_{1}\right)$ y erosión $\left(e_{2}\right)$, de profundidad efectiva $\left(s_{1}\right)$, textura $\left(s_{2}\right)$ y pedregosidad $\left(s_{3}\right)$, así como de drenaje (d) que va desde excesivo hasta moderadamente excesivo; no presentando limitaciones de densidad aparente.

Según se presenta en el Cuadro 2, dado que los criterios de selección de las prácticas para cada unidad de manejo se basaron en las limitantes reinantes en cada una de ellas, al momento de describirlas, se seleccionaron los sistemas agroforestales con aguacate, café y limón persa, considerando los requerimientos o exigencias de estos cultivos en cuanto a clima, suelo, altura y humedad, según Andújar et al. (2009), Féliz (2009)' y Rossó $(2009)^{2}$ respectivamente; pudiéndose indicar ciertas condicionantes para su instalación en las unidades

Cuadro 2. Descripción de las prácticas para el manejo de las tierras

\begin{tabular}{|c|c|c|c|c|c|c|c|c|c|c|}
\hline \multirow{2}{*}{ Descripción de las prácticas } & \multicolumn{9}{|c|}{ Unidades de manejo } & \multirow{2}{*}{ Condición } \\
\hline & MI & M2 & M3 & M4 & M5 & M6 & M7 & M8 & M9 & \\
\hline \multicolumn{11}{|l|}{ Prácticas conservacionistas } \\
\hline Protección & $x$ & $x$ & X & X & $x$ & $x$ & $x$ & $x$ & X & Sin condición \\
\hline Manejo forestal & $x$ & $x$ & $x$ & x & x & $x$ & $x$ & X & X & Sin condición \\
\hline Barbecho & $x$ & $x$ & $x$ & $x$ & $x$ & $x$ & $x$ & $x$ & $x$ & Sin condición \\
\hline \multicolumn{11}{|l|}{ Prácticas agroconservacionistas } \\
\hline Agroforestería con café & $x$ & & $x$ & & & $x$ & $x$ & $x$ & $x$ & $\begin{array}{l}\text { M7,M8,M9, solo en falda de la montaña, } \\
\text { cerca del arroyo. }\end{array}$ \\
\hline Agroforestería con aguacate & & $x$ & $x$ & & & $x$ & $x$ & & & $\begin{array}{l}\text { Solo en falda de la montaña, suelo con } \\
\text { rocas sueltas. }\end{array}$ \\
\hline Agroforestería con limón persa & $x$ & $x$ & $x$ & $x$ & x & $x$ & $x$ & $x$ & x & M3,M7,M8,9, solo en falda de la montaña. \\
\hline Abono orgánico sin estiércol & $x$ & $x$ & $x$ & $x$ & $x$ & $x$ & $x$ & $x$ & $x$ & M3,M7,M8,9, solo en falda de la montaña. \\
\hline Labranza mínima & $x$ & $x$ & & $x$ & $x$ & $x$ & $x$ & $x$ & $x$ & $\begin{array}{l}\text { M7,M8,M9, solo en falda de la montaña, } \\
\text { cerca del arroyo. }\end{array}$ \\
\hline Cobertura muerta & $x$ & $x$ & X & X & $x$ & $x$ & X & $x$ & X & Sin condición \\
\hline Abonos verdes & $x$ & $x$ & $x$ & $x$ & $x$ & $x$ & $x$ & $x$ & $x$ & Sin condición \\
\hline Rotación de cultivos & $x$ & $x$ & & $x$ & x & $x$ & $x$ & $x$ & $x$ & $\begin{array}{l}\text { M7,M8,M9, solo en falda de la montaña, } \\
\text { cerca del arroyo. }\end{array}$ \\
\hline Riego por goteo y microaspersión & $x$ & $x$ & & $x$ & x & $x$ & $x$ & $x$ & x & $\begin{array}{l}\text { M7,M8,M9, solo en falda de la montaña, } \\
\text { cerca del arroyo. }\end{array}$ \\
\hline \multicolumn{11}{|l|}{ Técnicas - conservación de suelos } \\
\hline Canal de guardia con barrera viva & $x$ & $x$ & $x$ & $x$ & $x$ & $x$ & $x$ & $x$ & $x$ & Sin condición \\
\hline Barrera muerta en contorno & $x$ & $x$ & $x$ & X & $x$ & $x$ & x & $x$ & X & Sin condición \\
\hline Barrera viva en contorno & $x$ & $x$ & $x$ & $x$ & $x$ & $x$ & $x$ & $x$ & x & Sin condición \\
\hline Terrazas individuales & $x$ & X & $x$ & $x$ & $x$ & $x$ & $x$ & $x$ & $x$ & Sin condición \\
\hline Readecuación de caminos & $x$ & $x$ & $x$ & $x$ & $x$ & $x$ & $x$ & $x$ & $x$ & Sin condición \\
\hline
\end{tabular}

Fuente: Creación propia.
I Féliz, Luis. II/ 2010. Técnico de CODOCAFE. Requerimientos del cultivo de café. (entrevista). Consejo Dominicano de Café (CODOCEFE). Barahona, República Dominicana.

2 Rossó, Héctor. I I/2000. Técnico Agrícola en la zona de Los Fríos. Requerimientos del cultivo de limón. (entrevista). Fundación Sur Futuro FSF. Padre Las Casas, República Dominicana. 
fisiográficas correspondientes; mientras que en las tierras de las unidades I, 4 y 5 no se recomienda la siembra de aguacates porque las limitaciones de fertilidad del suelo, textura, profundidad efectiva y drenaje respectivamente no les favorecen. De igual manera, no se recomienda la producción de café en las tierras de las unidades 2, 4 y 5 debido a las limitaciones de textura, fertilidad y profundidad efectiva que presentan.

De acuerdo con el mapa de capacidad de uso de las tierras, la mayor severidad dentro de las limitaciones de uso se muestra en las unidades fisiográficas (UF) $3,7,8$ y 9 , puesto que son las que tienen mayor vocación de protección y forestal, por lo que el desarrollo de la agricultura dentro de ellas debe hacerse aplicando ciertos controles de manejo. De igual manera, ante las mismas condiciones limitantes de las tierras, se han establecido prácticas conservacionistas y de protección de suelos sin condicionantes, ya que el efecto de su aplicación es siempre beneficioso para el sistema, aunque sus fines no sean productivos. Una de estas prácticas es la readecuación de caminos, cuyas estructuras son parte del sistema y su estado incide en las condiciones biofísicas y económicas de todo el entorno.

Una de las prácticas agroconservacionistas importante es el riego por goteo y microaspersión, considerando para su establecimiento las potencialidades que ofrece el arroyo Limón y otros existentes en la zona.

\section{Conclusiones}

Las tierras de la microcuenca El Limón abarcan descle la clase III hasta la clase VIII, todas mostrando limitaciones de erosión, suelo y drenaje.

Determinar la capacidad de uso de las tierras con base en las limitaciones que presentan las mismas es un elemento innovador en República Dominicana.

Las prácticas de manejo recomendadas obedecen a tales limitaciones, tomando en cuenta elementos biofísicos y de uso del suelo, siendo las principales las conservacionistas, agroconservacionistas y las técnicas de protección del suelo; este último es otro

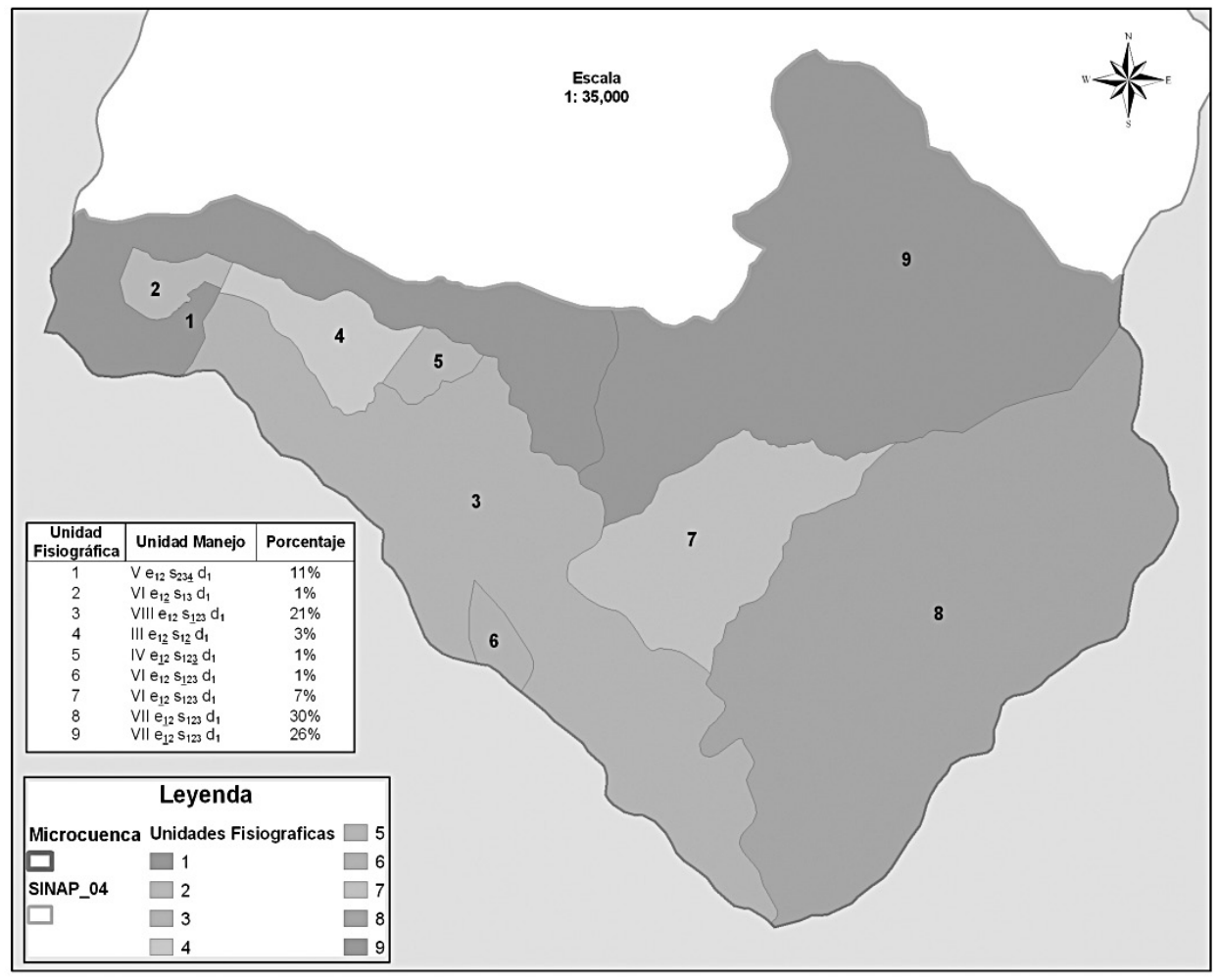

Figura 4. Mapa de capacidad de uso de las tierras.

Fuente: Creación propia. 
elemento innovador e importante para controlar el gran deterioro del sistema.

La mayor vocación de las tierras es de protección, manejo forestal y barbecho. Las prácticas agroconservacionistas deben desarrollarse en las zonas más bajas y cercanas al arroyo, con ciertas condicionantes.

La selección de los sistemas agroforestales de café y aguacate obedece a las limitaciones reinantes en las unidades de tierra donde se indica su aplicación, mientras que el uso del sistema con limón persa se recomienda en casi todas las tierras, lo cual significa que para la selección de los sistemas agroforestales hay que considerar, por un lado, las exigencias de los cultivos, y por otro, las limitaciones que presentan las tierras.

Se establecen sistemas de riego por goteo y microaspersión, considerando las limitantes de las tierras, así como las oportunidades que ofrecen el arroyo Limón y otros afluentes pequeños existentes en la zona.

\section{Bibliografía}

Andújar, D., Pérez, C. \& Jiménez, C. (2009). Manejo del cultivo del aguacate. Secretaría de Estado de Agricultura, Subsecretaría de Producción Agrícola. Santo Domingo, 15 p.

Cubero, D. (1996). Manual de manejo y conservación de suelos y aguas. San José: MAG, FAO, UNED, EUNED. 2da ed., 277 p.

Cubero, D. (2008). Determinación de la capacidad de uso de las tierras. Estudio de caso: Cuenca Jesús María. Costa Rica.

Gagnon, D. 2006. El Machete Verde. Manual Campesino de Conservación de Suelos. Managua: SUCO.

PROYECTA. (2007). Caracterización biofísica actualizada de las cuencas altas de la presa Sabana Yegua. Santo Domingo, $157 \mathrm{P}$.

Sistema de Diagnóstico de Tierras Tropicales. SOFTIERRA Inc. (200 I). San José, Costa Rica (Software). 